\title{
Monotone Iterative Technique for a Class of Slanted Cantilever Beam Equations
}

\author{
Mei Wei and Qiang Li \\ Department of Mathematics, Shanxi Normal University, Linfen 041000, China \\ Correspondence should be addressed to Qiang Li; lznwnuliqiang@126.com
}

Received 19 August 2017; Revised 1 December 2017; Accepted 13 December 2017; Published 31 December 2017

Academic Editor: Dan Simon

Copyright (C) 2017 Mei Wei and Qiang Li. This is an open access article distributed under the Creative Commons Attribution License, which permits unrestricted use, distribution, and reproduction in any medium, provided the original work is properly cited.

In this paper, we deal with the existence and uniqueness of the solutions of two-point boundary value problem of fourth-order ordinary differential equation: $u^{(4)}(t)=f\left(t, u(t), u^{\prime}(t)\right), t \in[0,1], u(0)=u^{\prime}(0)=u^{\prime \prime}(1)=u^{\prime \prime \prime}(1)=0$, where $f:[0,1] \times \mathbb{R}{ }^{2} \rightarrow \mathbb{R}$ is a continuous function. The problem describes the static deformation of an elastic beam whose left end-point is fixed and right is freed, which is called slanted cantilever beam. Under some weaker assumptions, we establish a new maximum principle by the perturbation of positive operator and construct the monotone iterative sequence of the lower and upper solutions, and, based on this, we obtain the existence and uniqueness results for the slanted cantilever beam.

\section{Introduction}

In mechanics, the two-point boundary value problems of fourth-order ordinary differential equations are mainly used to describe the static deformation of elastic beam under external force, and especially a model to study travelling waves in suspension bridges can be furnished by the fourthorder equation of nonlinearity. Due to the different support conditions of elastic beams, a variety of boundary value problems are derived; see [1].

In this paper, we deal with the existence and uniqueness results of solutions to the two-point boundary value problem of fourth-order ordinary differential equation

$$
\begin{gathered}
u^{(4)}(t)=f\left(t, u(t), u^{\prime}(t)\right), \quad t \in[0,1] \\
u(0)=u^{\prime}(0)=u^{\prime \prime}(1)=u^{\prime \prime \prime}(1)=0
\end{gathered}
$$

where $f:[0,1] \times \mathbb{R}^{2} \rightarrow \mathbb{R}$ is a continuous function. The problem is called slanted cantilever beam which describes the static deformation of an elastic beam whose left endpoint is fixed and right is freed. For the equation, the physical meaning of the first-order derivative $u^{\prime}(t)$ of unknown function is the slope, which reflects the curving degree of the elastic beam; see [1-5].
There are many results on the cantilever beam equation; see [4-17]. Specially, in [4, 14, 15], Agarwal et al. used the fixed point theorems of cone mapping to research the special case of BVP (1) that the nonlinear term $f$ does not contain the derivative term $u^{\prime}$, namely,

$$
\begin{aligned}
u^{(4)}(t) & =f(t, u(t)), \quad t \in[0,1], \\
u(0) & =u^{\prime}(0)=u^{\prime \prime}(1)=u^{\prime \prime \prime}(1)=0 .
\end{aligned}
$$

In these works, since there is no derivative term, the research about the solutions is simple and feasible relatively.

In $[6,7,10,16,17]$, for the fourth-order ordinary differential equation with the boundary value condition $u(0)=$ $u^{\prime}(0)=u^{\prime \prime}(1)=0, u^{\prime \prime \prime}(1)=\mu g(u(1))$, which means that the left end of the beam is fixed and the right is attached to a bearing device, the existence and multiplicity of solutions have been discussed by using the variational methods and critical point theory.

For the case of BVP (1), in [11], Yao constructed a successively iterative sequence by using the monotone iterative technique and applying the successively approximate method to prove an existence theorem. Recently, in [5], by using the fixed point index theory in cones, Li researched the existence of positive solutions of cantilever beam equation in which 
the nonlinear term contains all order derivatives of unknown function.

However, there are still many limitations in the study of this problem in recent years. First of all, most conclusions of the existences were obtained only by roughly estimating the properties of the corresponding Green function; secondly, most of the conditions for nonlinear term $f$ are very harsh, so the existence results of the solutions are not optimal.

For the solvability of elastic beam equations with other types of boundary conditions, many results have been obtained; see [18-24] and references therein. Specially, in [23], Li dealt the fourth-order boundary value problem

$$
\begin{array}{r}
u^{(4)}(t)=f\left(t, u(t), u^{\prime \prime}(t)\right), \quad t \in I, \\
u(0)=u(1)=u^{\prime \prime}(0)=u^{\prime \prime}(1)=0
\end{array}
$$

and obtained the existence and uniqueness of solutions by utilizing the perturbation of positive operator and the monotone iterative technique of upper and lower solutions. It is well known that the monotone iterative method of lower and upper solutions has been widely used in solving the boundary value problem of ordinary differential equations. However, as far as we know, no researchers studied BVP (1) by monotone iterative method of lower and upper solutions.

Motivated by the papers mentioned above, we will use the monotone iterative technique of lower and upper solutions to discuss the existence and uniqueness of BVP (1). It is well known that the theoretical basis of the monotone iterative technique is the maximum principle. It often requires two aspect of works for this method. One is to construct the iterative sequence and judge its monotonicity, and the other is to verify the convergence of the constructed sequence. Generally, For the case of BVP (2), the nonlinear item $f=f(t, u(t))$, if the linear differential operator at the left satisfies the maximum principle, then the monotone iterative technique is feasible; see [18-20]. However, in BVP (1), the nonlinear term contains the derivative; the general maximum principle cannot guarantee the monotonicity of the iterative sequence. Therefore, in order to ensure the feasibility of the monotone iterative technique, we should strengthen the maximum principle.

The purpose of this paper is to construct a new maximum principle for fourth-order differential operator

$$
L_{4} u=u^{(4)}+N u^{\prime}+M u,
$$

where $M, N$ are constants satisfying

$$
\frac{N}{2}+\frac{M}{3} \leq 1, \quad M, N \geq 0
$$

and establish the monotone iterative technique in the case of the lower and upper solutions existing in BVP (1). To the best of our knowledge, using this method to solve the problem of the solvability of cantilever beam equation is rare. It means that our conclusions are new and meaningful.

The paper is organized as follows. Section 2 provides the preliminary results which are used in theorems stated and proved in the article, and Section 3 presents the main results and its proof of the article.

\section{Preliminaries}

In this section, we introduce some basic concepts and preliminary facts which are used in this paper.

Let $I=[0,1], C(I)$, be a continuous function space endowed the maximum norm $\|u\|=\max _{t \in I}|u(t)|, C^{n}(I)(n=$ $1,2,3,4)$ are $n$-order continuous differentiable function spaces which are defined in $I$, and $C^{+}(I)$ denote a cone in the form of all nonnegative functions in $C(I)$. Evidently, $C^{+}(I)=$ $\{u \in C(I) \mid u(t) \geq 0, t \in I\}$.

Let constants $M, N$ satisfy the expression (5). In order to study the existence of solutions of the BVP (1), we establish a new maximum principle for the differential operator (4). To this end, we consider the corresponding fourth-order linear boundary value problem (LBVP)

$$
\begin{aligned}
& u^{(4)}(t)+N u^{\prime}(t)+M u(t)=h(t), \quad t \in I, \\
& u(0)=u^{\prime}(0)=u^{\prime \prime}(1)=u^{\prime \prime \prime}(1)=0 .
\end{aligned}
$$

Assume that $v(t)=u^{\prime}(t)$; then we have

$$
u(t)=\int_{0}^{t} v(s) d s:=T_{0} v(t) .
$$

Evidently, $\left\|T_{0}\right\|=1$. Therefore, the fourth-order LBVP (6) is equivalent to the following third-order boundary value problem:

$$
\begin{aligned}
v^{(3)}(t)+N v(t)+M T_{0} v(t) & =h(t), \quad t \in I, \\
v(0) & =v^{\prime}(1)=v^{\prime \prime}(1)=0 .
\end{aligned}
$$

We have known that, for any $h \in C(I)$, the third-order linear boundary value problem

$$
\begin{aligned}
v^{(3)}(t) & =h(t), \quad t \in I, \\
v(0) & =v^{\prime}(1)=v^{\prime \prime}(1)=0
\end{aligned}
$$

has a unique solution $v \in C^{3}(I)$, which can be expressed as

$$
v(t)=\int_{0}^{1} G(t, s) h(s) d s:=\operatorname{Sh}(t),
$$

where $G(t, s)$ is the Green function of LBVP (9) given by the following expressions:

$$
G(t, s)= \begin{cases}t s-\frac{1}{2} t^{2}, & 0 \leq t \leq s \leq 1, \\ \frac{1}{2} s^{2}, & 0 \leq s \leq t \leq 1 .\end{cases}
$$

Clearly, $G(t, s)$ is continuous, and the following lemma is established.

Lemma 1. $G(t, s)$ has the following properties:
(a) $G(t, s)>0$, for any $0<t, s<1$.
(b) $t G(s, s) \leq G(t, s) \leq \max _{t \in I} G(t, s)=G(s, s)$, for any $0 \leq t, s \leq 1$.
(c) $G(t, s) \leq s t$, for any $0 \leq t, s \leq 1$. 
Proof. From the expression of (11), it follows that (a) holds.

(b) For $0 \leq t \leq s \leq 1$, we have

$$
\begin{aligned}
G(t, s)-G(s, s) & =-\frac{1}{2}(s-t)^{2} \leq 0 \\
G(t, s)-t G(s, s) & =s t-\frac{1}{2} t^{2}-\frac{1}{2} t s^{2} \\
& =\frac{1}{2} t(s-t)+\frac{1}{2} s t(1-s) \geq 0 .
\end{aligned}
$$

For $0 \leq s \leq t \leq 1$

$$
G(t, s)=\frac{1}{2} s^{2}=G(s, s) \geq t G(s, s) .
$$

(c) From the expression (11), for any $t, s \in[0,1], G(t, s) \leq$ st holds obviously.

This completes the proof of Lemma 1.

From Lemma 1, we can obtain the following result which is needed in the proof of our main results.

Lemma 2. The solution operator $S: C(I) \rightarrow C(I)$ of $L B V P$ (9) is the completely continuous linear operator, and its norm satisfies $\|S\| \leq 1 / 6$. Furthermore, if $h \in C^{+}(I)$, then $\operatorname{Sh}(t) \geq$ $t\|S h\|$, for every $t \in I$.

Proof. From (10), we can easily obtain that the solution operator $S: C(I) \rightarrow C(I)$ of LBVP (9) is a completely continuous linear operator.

For any $h \in C(I)$, by (10) and (11), we obtain

$$
\begin{aligned}
|S h(t)| & \leq \int_{0}^{1} G(t, s) d s \cdot\|h\|=\frac{1}{6}\left(t^{3}-3 t^{2}+3 t\right)\|h\| \\
& \leq \frac{1}{6}\|h\|, \quad t \in I .
\end{aligned}
$$

It is easy to see that $\|S h\| \leq(1 / 6)\|h\|$, which implies that $\|S\| \leq$ $1 / 6$ holds.

Furthermore, for $h \in C^{+}(I)$, from (10) and the second inequality of Lemma $1(\mathrm{~b})$, we get

$$
|S h(t)| \leq \int_{0}^{1} G(s, s) h(s) d s, \quad t \in I .
$$

It follows that

$$
\|S h\| \leq \int_{0}^{1} G(s, s) h(s) d s .
$$

From Lemma 1(b), we have

$$
\begin{aligned}
S h(t) & =\int_{0}^{1} G(t, s) h(s) d s \geq t \int_{0}^{1} G(s, s) h(s) d s \\
& \geq t\|S h\| .
\end{aligned}
$$

This completes the proof of Lemma 2.

In order to establish the new maximum principle, we also need to prove the following lemma.
Lemma 3. Let there exist constants $M$ and $N$ satisfying the assumption (5); then LBVP (6) has a unique solution $u=T h \epsilon$ $C^{4}(I)$ for any $h \in C(I)$, and the solution operator $T: C(I) \rightarrow$ $C^{1}(I)$ is completely continuous. Specifically, when $h \in C^{+}(I)$, then the solution $u=T h$ satisfies $u(t) \geq 0, u^{\prime}(t) \geq 0, t \in I$.

Proof. According to the above analysis, if there exists the unique solution $v \in C^{3}(I)$ of $\operatorname{LBVP}(8)$, then $u=T_{0} v \in C^{4}(I)$ is the unique solution of LBVP (6). By the Lemma 2, LBVP (8) is equivalent to the operator equation

$$
\left(J+N S+M S T_{0}\right) v(t)=S h(t),
$$

where $J$ is the unit operator in $C(I)$. By Lemma 2, it follows that

$$
\begin{aligned}
\left\|N S+M S T_{0}\right\| & \leq N\|S\|+M\|S\| \cdot\left\|T_{0}\right\| \leq \frac{N}{6}+\frac{M}{6} \\
& \leq \frac{N}{4}+\frac{M}{6}=\frac{1}{2}\left(\frac{N}{2}+\frac{M}{3}\right) \leq \frac{1}{2} .
\end{aligned}
$$

Therefore, $J+N S+M S T_{0}$ creates bounded inverse operator. According to the Neumann expansion, we can obtain that

$$
\begin{aligned}
(J & \left.+N S+M S T_{0}\right)^{-1}=\sum_{n=0}^{\infty}(-1)^{n}\left(N S+M S T_{0}\right)^{n} \\
& =\sum_{n=0}^{\infty}(-1)^{n}\left(N S+M S T_{0}\right)^{2 n}\left(J-N S-M S T_{0}\right) ;
\end{aligned}
$$

its norm satisfies

$$
\left\|\left(J+N S+M S T_{0}\right)^{-1}\right\| \leq \frac{1}{1-\left\|\left(N S+M S T_{0}\right)\right\|} \leq 2 .
$$

Therefore, the operator equation (18) has a uniqueness solution

$$
v=\left(J+N S+M S T_{0}\right)^{-1} S h:=B h .
$$

Thus, LBVP (6) has the uniqueness solution $u=T_{0} v=$ $T_{0} B h:=T h$, where $B=\left(J+N S+M S T_{0}\right)^{-1} S, T=T_{0} B$. Since the operator $S: C(I) \rightarrow C(I)$ is completely continuous and $\left(J+N S+M S T_{0}\right)^{-1}$ is a bounded linear operator, then the operator $B: C(I) \rightarrow C(I)$ is completely continuous. Thus, according to the boundedness of $T_{0}: C(I) \rightarrow C^{1}(I)$, we can get that $T: C(I) \rightarrow C^{1}(I)$ is a completely continuous operator.

Now, we prove that, for any $h \in C^{+}(I)$, the solution $u=T h$ of LBVP (6) satisfies $u \geq 0, u^{\prime} \geq 0$.

Since $T_{0}$ and $S$ are the positive operators in $C(I)$, and $S h \leq$ $\|S h\|$, then from the definition of operator $T_{0}$, we have $T_{0} S h \leq$ $t\|S h\|$, and by Lemma 2(c), it is obvious that

$$
\begin{aligned}
& \left(N S+M S T_{0}\right) S h=(N S) S h+\left(M S T_{0}\right) S h \\
& \leq(N S(1)+M S(s))\|S h\| \\
& =\left(N \int_{0}^{1} H(t, s) d s+M \int_{0}^{1} s H(t, s) d s\right)\|S h\| \\
& \leq\left(\frac{N}{2} t+\frac{M}{3} t\right)\|S h\|=\left(\frac{N}{2}+\frac{M}{3}\right)\|S h\| t,
\end{aligned}
$$


for any $t \in I$. By Lemma 2, we can obtain

$$
\begin{aligned}
\left(J-N S-M S T_{0}\right) S h & =S h-\left(N S+M S T_{0}\right) S h \\
& \geq\|S h\| t-\left(\frac{N}{2}+\frac{M}{3}\right)\|S h\| t \\
& =\left(1-\left(\frac{N}{2}+\frac{M}{3}\right)\right)\|S h\| t \geq 0,
\end{aligned}
$$

for any $t \in I$. Since $T_{0}$ and $S$ are the positive operators, we can obtain that

$$
\begin{aligned}
B h & =\left(J+N S+M S T_{0}\right)^{-1} S h \\
& =\sum_{n=0}^{\infty}\left(N S+M S T_{0}\right)^{2 n}\left(J-N S-M S T_{0}\right) S h \geq 0 .
\end{aligned}
$$

Therefore, the solution of $\operatorname{LBVP}(6)$ satisfies $u=T_{0} B h \geq 0$, and $u^{\prime}=B h \geq 0$. This completes the proof of Lemma 3 .

According to the conclusion of Lemma 3, the following maximum principle can be obtained.

Lemma 4. Let there exist constants $M$ and $N$ satisfying the assumption (5), if $u \in C^{4}(I)$ satisfies

$$
\begin{aligned}
u^{(4)}(t)+N u^{\prime}(t)+M u(t) & \geq 0, \quad t \in I, \\
u(0) & \geq 0, \\
u^{\prime}(0) & \geq 0 \\
u^{\prime \prime}(1) & \geq 0 \\
u^{\prime \prime \prime}(1) & \leq 0
\end{aligned}
$$

then $u(t) \geq 0, u^{\prime}(t) \geq 0$ for any $t \in I$.

\section{Main Results}

Now, we are in the position to state and prove our main results. We will apply monotone iterative method of the lower and upper solutions to obtain the existence and uniqueness of solutions for cantilever beam equation (1). To this end, we define the lower and upper solutions of BVP (1).

Definition 5. If $\alpha(t) \in C^{4}(I)$ satisfies

$$
\begin{aligned}
\alpha^{(4)}(t) & \leq f\left(t, \alpha(t), \alpha^{\prime}(t)\right), \quad t \in I, \\
\alpha(0) & \leq 0 \\
\alpha^{\prime}(0) & \leq 0 \\
\alpha^{\prime \prime}(1) & \leq 0 \\
\alpha^{\prime \prime \prime}(1) & \geq 0
\end{aligned}
$$

then $\alpha(t)$ is called a lower solution of BVP (1). If the inequality of (27) is inverse, then $\alpha(t)$ is called an upper solution of BVP (1).
Theorem 6. Let $f: I \times \mathbb{R} \times \mathbb{R} \rightarrow \mathbb{R}$ be continuous, and there are lower and upper solutions $\alpha$ and $\beta$ for BVP (1), satisfying $\alpha \leq \beta, \alpha^{\prime} \leq \beta^{\prime}$. If $f$ satisfies the following condition:

(F1) there exist positive constants $M$ and $N$ satisfying (5), such that

$$
\begin{aligned}
& f\left(t, u_{2}, v_{2}\right)-f\left(t, u_{1}, v_{1}\right) \\
& \quad \geq-M\left(u_{2}-u_{1}\right)-N\left(v_{2}-v_{1}\right),
\end{aligned}
$$

for arbitrary $t \in I, u_{1}, u_{2} \in[\alpha, \beta], v_{1}, v_{2} \in$ $\left[\alpha^{\prime}, \beta^{\prime}\right], u_{2} \geq u_{1}, v_{2} \geq v_{1}$,

then $B V P(1)$ has one maximal solution $\bar{u}$ and minimal solution $\underline{u}$ between $\alpha$ and $\beta$.

Proof. Let $D=\left\{u \in C^{1}(I) \mid \alpha \leq u \leq \beta, \alpha^{\prime} \leq u^{\prime} \leq \beta^{\prime}\right\}$, Clearly, $D$ is a bounded nonempty convex closed set in $C^{1}(I)$. follows:

For any $u \in D$, we define an operator $F: D \rightarrow C(I)$ as

$$
F(u)(t)=f\left(t, u(t), u^{\prime}(t)\right)+N u^{\prime}(t)+M u(t) .
$$

According to the continuity of $f$, it is easy to see that $F$ is the continuous bounded operator in $C(I)$. Let $T$ be the solution operator of LBVP (6); then the solution of BVP (1) in $D$ is equivalent to the fixed point of the composition operator $Q=$ $T \circ F: D \rightarrow C^{1}(I)$. We can easily obtain that operator $Q$ as completely continuous by the complete continuity of $T$ and the boundedness of $F$. In the following, we will take four steps to prove the conclusion.

Step 1. We prove that $Q(D) \subset D$.

To this end, we let $x=Q u$ for every $u \in D$. And define $h=F(u)$, and then $x=T h$ is the solution of LBVP (6). Thus, $x \in C^{4}(I)$ satisfy

$$
\begin{aligned}
& x^{(4)}(t)+N x^{\prime}(t)+M x(t) \\
& \quad=f\left(t, u(t), u^{\prime}(t)\right)+N u^{\prime}(t)+M u(t), \quad t \in I, \\
& x(0)=x^{\prime}(0)=x^{\prime \prime}(1)=x^{\prime \prime \prime}(1)=0 .
\end{aligned}
$$

Then by the definition of the lower and upper solutions and the assumption (F1), it is clear that

$$
\begin{aligned}
&(x-\alpha)^{(4)}+N(x-\alpha)^{\prime}+M(x-\alpha) \\
& \geq f\left(t, u, u^{\prime}\right)-f\left(t, \alpha, \alpha^{\prime}\right)+N(u-\alpha)^{\prime} \\
&+M(u-\alpha) \\
& \geq-M(u-\alpha)-N(u-\alpha)^{\prime}+N(u-\alpha)^{\prime} \\
&+M(u-\alpha)=0, \quad \forall t \in I, \\
&(\beta-x)^{(4)}+N(\beta-x)^{\prime}+M(\beta-x) \\
& \geq f\left(t, \beta, \beta^{\prime}\right)-f\left(t, u, u^{\prime}\right)+N(\beta-u)^{\prime} \\
&+M(\beta-u)
\end{aligned}
$$




$$
\begin{aligned}
\geq & -M(\beta-u)-N(\beta-u)^{\prime}+N(\beta-u)^{\prime} \\
& +M(\beta-u)=0, \quad \forall t \in I .
\end{aligned}
$$

By the boundary conditions, we can get that

$$
\begin{gathered}
(x-\alpha)(0) \geq 0, \\
(x-\alpha)^{\prime}(0) \geq 0, \\
(x-\alpha)^{\prime \prime}(1) \geq 0, \\
(x-\alpha)^{\prime \prime \prime}(1) \leq 0, \\
(\beta-x)(0) \geq 0, \\
(x-\alpha)^{\prime}(0) \geq 0, \\
(x-\alpha)^{\prime \prime}(1) \geq 0, \\
(x-\alpha)^{\prime \prime \prime}(1) \leq 0 .
\end{gathered}
$$

Applying Lemma (10) to $x-\alpha$ and $\beta-x$, we have

$$
\begin{gathered}
x-\alpha \geq 0, \\
(x-\alpha)^{\prime} \geq 0 ; \\
\beta-x \geq 0, \\
(\beta-x)^{\prime} \geq 0,
\end{gathered}
$$

which means $\alpha \leq x \leq \beta, \alpha^{\prime} \leq x^{\prime} \leq \beta^{\prime}$ in $I$. Therefore, we can conclude that $Q(D) \subset D$.

Step 2. We show that if $u_{1}, u_{2} \in D$ satisfy $\alpha \leq u_{1} \leq u_{2} \leq$ $\beta, \alpha^{\prime} \leq u_{1}^{\prime} \leq u_{2}^{\prime} \leq \beta^{\prime}$, then $Q u_{1} \leq Q u_{2},\left(Q u_{1}\right)^{\prime} \leq\left(Q u_{2}\right)^{\prime}$ holds.

In fact, similar to the first step, let $x_{1}=Q u_{1}, x_{2}=Q u_{2}$, and then by the assumption (F1), we can obtain

$$
\begin{aligned}
\left(x_{2}-x_{1}\right)^{4}+N\left(x_{2}-x_{1}\right)^{\prime}+M\left(x_{2}-x_{1}\right) \\
=f\left(t, u_{2}, u_{2}^{\prime}\right)-f\left(t, u_{1}, u_{1}^{\prime}\right)+N\left(u_{2}^{\prime}-u_{1}^{\prime}\right) \\
\quad+M\left(u_{2}-u_{1}\right) \\
\quad-M\left(u_{2}-u_{1}\right)-N\left(u_{2}^{\prime}-u_{1}^{\prime}\right)+N\left(u_{2}^{\prime}-u_{1}^{\prime}\right) \\
\quad+M\left(u_{2}-u_{1}\right) \geq 0, \quad \forall t \in I .
\end{aligned}
$$

By the boundary conditions, we can get that

$$
\begin{gathered}
\left(x_{2}-x_{1}\right)(0) \geq 0, \\
\left(x_{2}-x_{1}\right)^{\prime}(0) \geq 0, \\
\left(x_{2}-x_{1}\right)^{\prime \prime}(1) \geq 0, \\
\left(x_{2}-x_{1}\right)^{\prime \prime \prime}(1) \leq 0
\end{gathered}
$$

then applying Lemma (10) to $x_{2}-x_{1}$, we have

$$
\begin{array}{r}
x_{2}-x_{1} \geq 0, \\
\left(x_{2}-x_{1}\right)^{\prime} \geq 0,
\end{array}
$$

$\forall t \in I$

which means that $Q u_{2} \geq A u_{1},\left(Q u_{2}\right)^{\prime} \geq\left(Q u_{1}\right)^{\prime}$.

Step 3. We demonstrate that there exist solutions between $\alpha$ and $\beta$.

We use $\alpha$ and $\beta$ as the initial element for constructing iterative sequence

$$
\begin{aligned}
& \alpha_{n}=Q \alpha_{n-1}, \\
& \beta_{n}=Q \beta_{n-1}, \\
& \quad n=1,2, \ldots
\end{aligned}
$$

According to the definition of the operator Q, Steps 1 and 2 , we can easily see that

$$
\begin{aligned}
& \alpha_{0} \leq \alpha_{1} \leq \cdots \leq \alpha_{n} \leq \beta_{n} \leq \cdots \leq \beta_{1} \leq \beta_{0}, \\
& \alpha_{0}^{\prime} \leq \alpha_{1}^{\prime} \leq \cdots \leq \alpha_{n}^{\prime} \leq \beta_{n}^{\prime} \leq \cdots \leq \beta_{1}^{\prime} \leq \beta_{0}^{\prime},
\end{aligned}
$$

which means that $\left\{\alpha_{n}\right\}$ and $\left\{\beta_{n}\right\}$ are monotone increasing and decreasing in the order interval $[\alpha, \beta]$, respectively; $\left\{\alpha_{n}^{\prime}\right\}$ and $\left\{\beta_{n}^{\prime}\right\}$ are also monotonous in the order interval $\left[\alpha^{\prime}, \beta^{\prime}\right]$.

By the compactness of $Q$, we know that $\left\{\alpha_{n}\right\},\left\{\beta_{n}\right\} \quad C$ $Q(D)$ are the relatively compact sets in $C^{1}(I)$, and, therefore, they have the uniformly convergent subsequence in $C^{1}(I)$. Then by (38), $\left\{\alpha_{n}\right\},\left\{\beta_{n}\right\},\left\{\alpha_{n}^{\prime}\right\},\left\{\beta_{n}^{\prime}\right\}$ are all uniformly convergent in $I$; therefore, $\left\{\alpha_{n}\right\}$ and $\left\{\beta_{n}\right\}$ are uniformly convergent in $C^{1}(I)$, which means there exist $\underline{u}$ and $\bar{u} \in C^{1}(I)$, such that $\alpha_{n} \rightarrow \underline{u}, \beta_{n} \rightarrow \bar{u}$. Since $D$ is a convex closed set in $C^{1}(I)$, it is obvious that $\underline{u}, \bar{u} \in D$. In the expression (37), we let $n \rightarrow \infty$, and then, from the continuity of $Q$, we can easily see $\underline{u}=Q \underline{u}, \bar{u}=Q \bar{u}$, for any $t \in I$. Thus, $\underline{u}$ and $\bar{u}$ are the solutions of BVP (1) between $\alpha$ and $\beta$.

Step 4 . We testify that $\underline{u}$ and $\bar{u}$ are the minimal and maximal solutions of BVP (1) between $\alpha$ and $\beta$, respectively.

Let $u \in D$ be an arbitrary solution of $\operatorname{LBVP}(6)$; then $u(t)$ satisfies

$$
\begin{gathered}
\alpha \leq u \leq \beta \\
\alpha^{\prime} \leq u^{\prime} \leq \beta^{\prime} .
\end{gathered}
$$

By Step 2, using $Q$ acting $n$ times for the last expression, it can be easily obtained that

$$
\begin{aligned}
& \alpha_{n} \leq u \leq \beta_{n}, \\
& \alpha_{n}^{\prime} \leq u^{\prime} \leq \beta_{n}^{\prime}, \\
& \quad n=1,2, \ldots .
\end{aligned}
$$


Taking $n \rightarrow \infty$, we can see

$$
\begin{aligned}
& \underline{u} \leq u \leq \bar{u}, \\
& \underline{u}^{\prime} \leq u^{\prime} \leq \bar{u}^{\prime} .
\end{aligned}
$$

It can be easily obtained that $\underline{u}$ and $\bar{u}$ are the minimum and maximum solutions of BVP (1) between $\alpha$ and $\beta$, respectively.

This completes the proof of Theorem 6.

From the above proof process, the next corollary can be easily obtained.

Corollary 7. Let $f: I \times \mathbb{R} \times \mathbb{R} \rightarrow \mathbb{R}$ be continuous, and there exist lower and upper solutions $\alpha$ and $\beta$ for BVP (1), satisfying $\alpha \leq \beta, \alpha^{\prime} \leq \beta^{\prime}$. If $f$ satisfies the assumption (F1), we use $\alpha$ and $\beta$ as the initial elements to construct iterative sequences $\left\{\alpha_{n}\right\}$ and $\left\{\beta_{n}\right\}$ by linear iterative equation

$$
\begin{aligned}
& u_{n}^{(4)}(t)+N u_{n}^{\prime}+M u_{n} \\
& =f\left(t, u_{n-1}(t), u_{n-1}^{\prime}(t)\right)+N u_{n-1}^{\prime}+M u_{n-1}, \\
& u_{n}(0)=u_{n}^{\prime}(0)=u_{n}^{\prime \prime}(1)=u_{n}^{\prime \prime \prime}(1)=0 ;
\end{aligned}
$$

then we can obtain that

$$
\begin{aligned}
& \lim _{n \rightarrow \infty} \alpha_{n}(t)=\underline{u}(t), \\
& \lim _{n \rightarrow \infty} \beta_{n}(t)=\bar{u}(t), \\
& \lim _{n \rightarrow \infty} \alpha_{n}^{\prime}(t)=\underline{u}^{\prime}(t), \\
& \lim _{n \rightarrow \infty} \beta_{n}^{\prime}(t)=\underline{u}(t)
\end{aligned}
$$

uniformly hold for arbitrary $t \in I$, where $\underline{u}$ and $\bar{u}$ are the minimal and maximal solutions of BVP (1) in the set

$$
D=\left\{u \in C^{1}(I) \mid \alpha \leq u \leq \beta, \alpha^{\prime} \leq u^{\prime} \leq \beta^{\prime}\right\},
$$

respectively.

Theorem 6 gives the existence of the solution of BVP (1). Now, we can further discuss the uniqueness result of the solutions by strengthening the assumption (F1).

Theorem 8. Let $f: I \times \mathbb{R} \times \mathbb{R} \rightarrow \mathbb{R}$ be continuous, and there exist lower and upper solutions $\alpha$ and $\beta$ for BVP (1), satisfying $\alpha \leq \beta, \alpha^{\prime} \leq \beta^{\prime}$. If $f$ satisfies the assumption (F1) and the following condition:

(F2) there exist positive constants $C_{1}$ and $C_{2}$ satisfying

$$
C_{1}+C_{2}+M+N<3
$$

such that

$$
\begin{aligned}
& f\left(t, u_{2}, v_{2}\right)-f\left(t, u_{1}, v_{1}\right) \\
& \quad \leq C_{1}\left(u_{2}-u_{1}\right)+C_{2}\left(v_{2}-v_{1}\right)
\end{aligned}
$$

for every $t \in I, u_{1}, u_{2} \in D, v_{1}, v_{2} \in\left[\alpha^{\prime}, \beta^{\prime}\right], u_{2} \geq$ $u_{1}, v_{2} \geq v_{1}$, then BVP (1) has a unique solution $u^{*}$ in $D$, and, for every $u_{0} \in D$, the monotone iterative sequence $u_{n}$ constructed by (42) uniformly converges to the unique solution $u^{*}$.

Proof. By the proof of Theorem 6, when the assumption (F1) holds, then the BVP (1) has maximal solution $\bar{u}$ and minimal solution $\underline{u}$ in $D$, and for every solution $u \in D$, we have $\underline{u} \leq$ $u \leq \bar{u}, \underline{u}^{\prime} \leq u^{\prime} \leq \bar{u}^{\prime}$. Next, we need to prove that $\underline{u}=\bar{u}$.

According to the proof of Lemma (9), the operator $B$ : $C(I) \rightarrow C(I)$ defined by (22) is a positive linear operator, and its norm satisfies

$$
\|B\| \leq\left\|\left(J+N S+M S T_{0}\right)^{-1}\right\| \cdot\|S\| \leq 2 \times \frac{1}{6}=\frac{1}{3} .
$$

Since $T=T_{0} B$, for any $h \in C(I)$, we have $(T h)^{\prime}=B h$.

Assuming that $\left\{\alpha_{n}\right\},\left\{\beta_{n}\right\}$ are the monotone iterative sequences constructed in Theorem 6 , by the assumptions (F1) and (F2) and the positivity of operator $B$, we can see

$$
\begin{aligned}
\beta_{n}^{\prime} & -\alpha_{n}^{\prime}=\left(Q \beta_{n-1}\right)^{\prime}-\left(Q \alpha_{n-1}\right)^{\prime}=\left[T\left(F\left(\beta_{n-1}\right)\right)\right]^{\prime} \\
& -\left[T\left(F\left(\alpha_{n-1}\right)\right)\right]^{\prime}=B\left(F\left(\beta_{n-1}\right)\right)-B\left(F\left(\alpha_{n-1}\right)\right) \\
& =B\left(F\left(\beta_{n-1}\right)-F\left(\alpha_{n-1}\right)\right)=B\left[f\left(t, \beta_{n-1}, \beta_{n-1}^{\prime}\right)\right. \\
& -f\left(t, \alpha_{n-1}, \alpha_{n-1}^{\prime}\right)+M\left(\beta_{n-1}-\alpha_{n-1}\right) \\
& \left.+N\left(\beta_{n-1}^{\prime}-\alpha_{n-1}^{\prime}\right)\right] \leq B\left[\left(C_{1}+M\right)\left(\beta_{n-1}-\alpha_{n-1}\right)\right. \\
& \left.+\left(C_{2}+N\right)\left(\beta_{n-1}^{\prime}-\alpha_{n-1}^{\prime}\right)\right]=\left(C_{1}+M\right) B T_{0}\left(\beta_{n-1}^{\prime}\right. \\
& \left.-\alpha_{n-1}^{\prime}\right)+\left(C_{2}+N\right) B\left(\beta_{n-1}^{\prime}-\alpha_{n-1}^{\prime}\right) \\
& =\left(\left(C_{1}+M\right) B T_{0}+\left(C_{2}+N\right) B\right)\left(\beta_{n-1}^{\prime}-\alpha_{n-1}^{\prime}\right)
\end{aligned}
$$

which implies that

$$
\begin{aligned}
\left\|\beta_{n}^{\prime}-\alpha_{n}^{\prime}\right\| \leq & \left(\left(C_{1}+M\right)\left\|T_{0}\right\|+\left(C_{2}+N\right)\right)\|B\| \\
& \cdot\left\|\beta_{n-1}^{\prime}-\alpha_{n-1}^{\prime}\right\| \\
\leq & \left(C_{1}+C_{2}+M+N\right)\|B\| \cdot\left\|\beta_{n-1}^{\prime}-\alpha_{n-1}^{\prime}\right\| .
\end{aligned}
$$

Therefore, we can get

$$
\begin{aligned}
\left\|\beta_{n}^{\prime}-\alpha_{n}^{\prime}\right\| \leq & \left(C_{1}+C_{2}+M+N\right)\|B\| \cdot\left\|\beta_{n-1}^{\prime}-\alpha_{n-1}^{\prime}\right\| \\
\leq & \left(C_{1}+C_{2}+M+N\right)^{2}\|B\|^{2} \\
& \cdot\left\|\beta_{n-2}^{\prime}-\alpha_{n-2}^{\prime}\right\| \leq \cdots \\
\leq & \left(C_{1}+C_{2}+M+N\right)^{n}\|B\|^{n} \cdot\left\|\beta_{0}^{\prime}-\alpha_{0}^{\prime}\right\| .
\end{aligned}
$$

By (47) and the assumption (F2), we have

$$
\begin{array}{r}
\left\|\beta_{n}^{\prime}-\alpha_{n}^{\prime}\right\| \leq\left(\frac{C_{1}+C_{2}+M+N}{3}\right)^{n} \cdot\left\|\beta_{0}^{\prime}-\alpha_{0}^{\prime}\right\| \\
(n \longrightarrow 0 \\
(n \rightarrow \infty) .
\end{array}
$$


Thus, we have

$$
\begin{array}{r}
\left\|\beta_{n}-\alpha_{n}\right\|=\left\|T_{0}\left(\beta_{n}^{\prime}-\alpha_{n}^{\prime}\right)\right\| \leq\left\|T_{0}\right\| \cdot\left\|\beta_{n}^{\prime}-\alpha_{n}^{\prime}\right\| \longrightarrow 0 \\
(n \longrightarrow \infty) .
\end{array}
$$

Therefore, by the conclusions of Corollary 7, we can obtain

$$
\underline{u}=\lim _{n \rightarrow \infty} \alpha_{n}=\lim _{n \rightarrow \infty} \beta_{n}=\bar{u} .
$$

Consequently, $u^{*}=\underline{u}=\bar{u}$ is the unique solution of BVP (1).

Now, we need to testify that the monotone iterative sequence $u_{n}$ constructed by (42) uniformly converges to the unique solution $u^{*}$.

Assuming $u_{0} \in D$, then the monotone iterative sequence $u_{n}$ used $u_{0}$ as the initial element constructed by (42) satisfying $u_{n}=A u_{n-1}, n=1,2, \ldots$ According to Step 2 of the proof process of Theorem 6 , it is easy to see that

$$
\begin{aligned}
& \alpha_{n} \leq u_{n} \leq \beta_{n}, \\
& \alpha_{n}^{\prime} \leq u_{n}^{\prime} \leq \beta_{n}^{\prime}, \\
& \quad n=0,1,2, \ldots .
\end{aligned}
$$

Taking $n \rightarrow \infty$, it follows that $u_{n} \rightarrow u^{*}$ in $C^{1}(I)$. Therefore, the conclusion is established.

Finally, we give a numerical example to illustrate our theoretical results.

Example 1. Consider the following nonlinear problem:

$$
\begin{aligned}
u^{(4)}(t)=\frac{1}{3} \sin t \cdot u(t)+\frac{1}{3} \cos t \cdot u^{\prime}(t)+\frac{1}{2} e^{t}, & \\
t & \in[0,1],
\end{aligned}
$$

$$
u(0)=u^{\prime}(0)=u^{\prime \prime}(1)=u^{\prime \prime \prime}(1)=0 .
$$

Clearly, $\alpha(t) \equiv 0$ is a lower solution of problem (55). Letting $\beta(t)=e^{t}$, we can obtain that

$$
\begin{aligned}
& \frac{1}{3} \sin t \cdot \beta(t)+\frac{1}{3} \cos t \cdot \beta^{\prime}(t)+\frac{1}{2} e^{t} \\
& \leq \frac{\sqrt{2}}{3} \sin \left(t+\frac{\pi}{4}\right) e^{t}+\frac{1}{2} e^{t} \leq\left(\frac{\sqrt{2}}{3}+\frac{1}{2}\right) e^{t} \leq e^{t} \\
& =\beta^{(4)}(t) ;
\end{aligned}
$$

it is means that $\beta(t)=e^{t}$ is a upper solution of problem (55).

On the other hand, for arbitrary $t \in[0,1]$, when $u_{1}, u_{2} \in$ $\left[0, e^{t}\right], v_{1}, v_{2} \in\left[0, e^{t}\right]$, and $u_{2} \geq u_{1}, v_{2} \geq v_{1}$, we can easily obtain

$$
\begin{aligned}
& \left(\frac{1}{3} \sin t \cdot u_{2}(t)+\frac{1}{3} \cos t \cdot v_{2}(t)\right) \\
& -\left(\frac{1}{3} \sin t \cdot u_{1}(t)+\frac{1}{3} \cos t \cdot v_{1}(t)\right) \\
& \quad=\frac{1}{3} \sin t\left(u_{2}(t)-u_{1}(t)\right)+\frac{1}{3} \cos t\left(v_{2}(t)-v_{1}(t)\right) \\
& \quad \geq-\frac{1}{3}\left(u_{2}(t)-u_{1}(t)\right)-\frac{1}{3}\left(v_{2}(t)-v_{1}(t)\right)
\end{aligned}
$$

which implies that

$$
\begin{aligned}
f\left(t, u(t), u^{\prime}(t)\right)= & \frac{1}{3} \sin t \cdot u(t)+\frac{1}{3} \cos t \cdot u^{\prime}(t) \\
& +\frac{1}{2} e^{t}
\end{aligned}
$$

satisfies the condition (F1) for $M=N=1 / 3$. Then, by Theorem 6, problem (55) has at least one maximal solution $\bar{u}$ and minimal solution $u$ between 0 and $e^{t}$.

Furthermore, it is obvious that

$$
\begin{gathered}
\frac{1}{3} \sin t\left(u_{2}(t)-u_{1}(t)\right)+\frac{1}{3} \cos t\left(v_{2}(t)-v_{1}(t)\right) \\
\leq \frac{1}{3}\left(u_{2}(t)-u_{1}(t)\right)+\frac{1}{3}\left(v_{2}(t)-v_{1}(t)\right),
\end{gathered}
$$

which implies that $f\left(t, u(t), u^{\prime}(t)\right)$ satisfies the condition (F2) for $C_{1}=C_{2}=M=N=1 / 3$. Then, by Theorem 8, the problem (55) has a unique solution $u^{*}$ which satisfies $0 \leq u^{*} \leq e^{t}, 0 \leq\left(u^{*}\right)^{\prime} \leq e^{t}$.

\section{Conflicts of Interest}

The authors declare that they have no conflicts of interest.

\section{References}

[1] C. P. Gupta, "Existence and uniqueness theorems for the bending of an elastic beam equation," Applicable Analysis: An International Journal, vol. 26, no. 4, pp. 289-304, 1988.

[2] A. R. Aftabizadeh, "Existence and uniqueness theorems for fourth-order boundary value problems," Journal of Mathematical Analysis and Applications, vol. 116, no. 2, pp. 415-426, 1986.

[3] C. P. Gupta, "Existence and uniqueness results for the bending of an elastic beam equation at resonance," Journal of Mathematical Analysis and Applications, vol. 135, no. 1, pp. 208-225, 1988.

[4] R. P. Agarwal and D. O'Regan, "Multiplicity results for singular conjugate, focal, and $(n, p)$ problems," Journal of Differential Equations, vol. 170, no. 1, pp. 142-156, 2001.

[5] Y. Li, "Existence of positive solutions for the cantilever beam equations with fully nonlinear terms," Nonlinear Analysis: Real World Applications, vol. 27, pp. 221-237, 2016.

[6] A. Cabada and S. Tersian, "Multiplicity of solutions of a two point boundary value problem for a fourth-order equation," Applied Mathematics and Computation, vol. 219, no. 10, pp. 52615267, 2013.

[7] A. Hadjian and M. Ramezani, "Existence of infinitely many solutions for fourth-order equations depending on two parameters," Electronic Journal of Differential Equations, vol. 117, pp. 1-10, 2017.

[8] D. R. Anderson and J. Hoffacker, "Existence of solutions for a cantilever beam problem," Journal of Mathematical Analysis and Applications, vol. 323, no. 2, pp. 958-973, 2006.

[9] Q. A. Dang and T. K. Ngo, "Existence results and iterative method for solving the cantilever beam equation with fully nonlinear term," Nonlinear Analysis: Real World Applications, vol. 36, pp. 56-68, 2017.

[10] G. Bonanno, A. Chinnì, and S. A. Tersian, "Existence results for a two point boundary value problem involving a fourth-order equation," Electronic Journal of Qualitative Theory of Differential Equations, vol. 2015, pp. 1-9, 2015. 
[11] Q. Yao, "Monotonically iterative method of nonlinear cantilever beam equations," Applied Mathematics and Computation, vol. 205, no. 1, pp. 432-437, 2008.

[12] Q. Yao, "Solvability of singular cantilever beam equation," Annals of Differential Equations. Weifen Fangcheng Niankan, vol. 24, no. 1, pp. 93-99, 2008.

[13] Q. Yao, "Local existence of multiple positive solutions to a singular cantilever beam equation," Journal of Mathematical Analysis and Applications, vol. 363, no. 1, pp. 138-154, 2010.

[14] R. P. Agarwal and D. O'Regan, "Twin solutions to singular boundary value problems," Proceedings of the American Mathematical Society, vol. 128, no. 7, pp. 2085-2094, 2000.

[15] R. P. Agarwal, D. O’Regan, and V. Lakshmikantham, "Singular $(p, n-p)$ focal and $(n, p)$ higher order boundary value problems," Nonlinear Analysis. Theory, Methods \& Applications. An International Multidisciplinary Journal, vol. 42, no. 2, Ser. A: Theory Methods, pp. 215-228, 2000.

[16] L. Yang, H. Chen, and X. Yang, "The multiplicity of solutions for fourth-order equations generated from a boundary condition," Applied Mathematics Letters, vol. 24, no. 9, pp. 1599-1603, 2011.

[17] Y. Song, "A nonlinear boundary value problem for fourth-order elastic beam equations," Tijdschrift voor Urologie, vol. 2014, no. 1, pp. 1-11, 2014.

[18] A. Cabada, "The method of lower and upper solutions for second, third, fourth, and higher order boundary value problems," Journal of Mathematical Analysis and Applications, vol. 185, no. 2, pp. 302-320, 1994.

[19] A. Cabada, J. A. Cid, and L. s. Sanchez, "Positivity and lower and upper solutions for fourth order boundary value problems," Nonlinear Analysis: Theory, Methods \& Applications, vol. 67, no. 5, pp. 1599-1612, 2007.

[20] C. De Coster and L. Sanchez, "Upper and lower solutions, ambrosetti-prodi problem and positive solutions for fourth order O.D.E," Rivista di Matemàtica Pura ed Applicata, no. 14, pp. 57-82, 1994.

[21] R. Ma, J. Zhang, and S. Fu, "The method, of lower and upper solutions for fourth-order two-point boundary value problems," Journal of Mathematical Analysis and Applications, vol. 215, pp. 415-422, 1997.

[22] Y. S. Yang, "Fourth-order two-point boundary value problems," Proceedings of the American Mathematical Society, vol. 104, no. 1, pp. 175-180, 1988.

[23] Y. Li, "A monotone iterative technique for solving the bending elastic beam equations," Applied Mathematics and Computation, vol. 217, no. 5, pp. 2200-2208, 2010.

[24] Z. Bai, "The upper and lower solution method for some fourthorder boundary value problems," Nonlinear Analysis: Theory, Methods \& Applications, vol. 67, no. 6, pp. 1704-1709, 2007. 


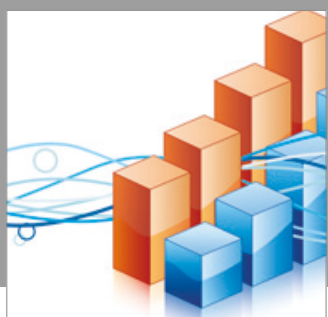

Advances in

Operations Research

vatersals

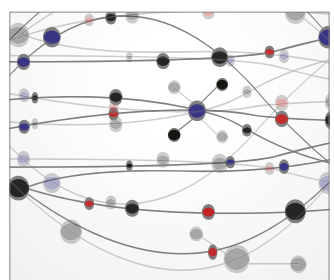

\section{The Scientific} World Journal
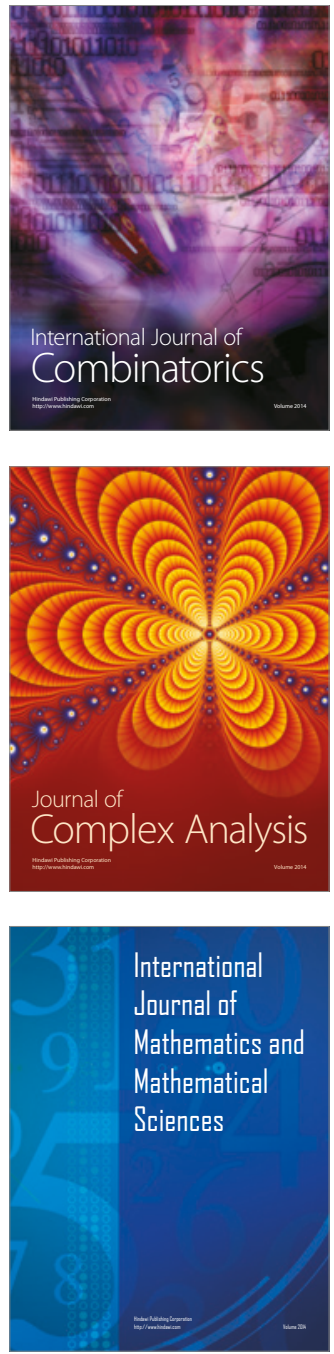
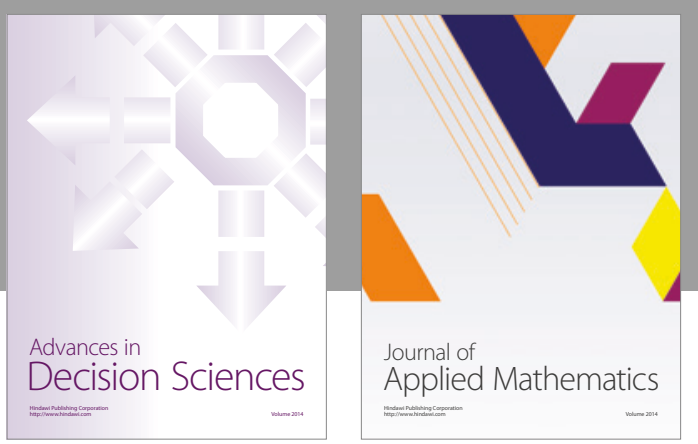

Algebra

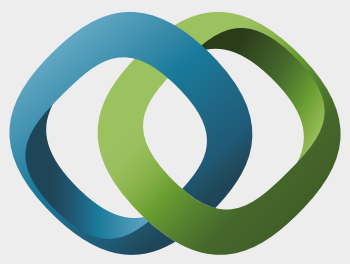

\section{Hindawi}

Submit your manuscripts at

https://www.hindawi.com
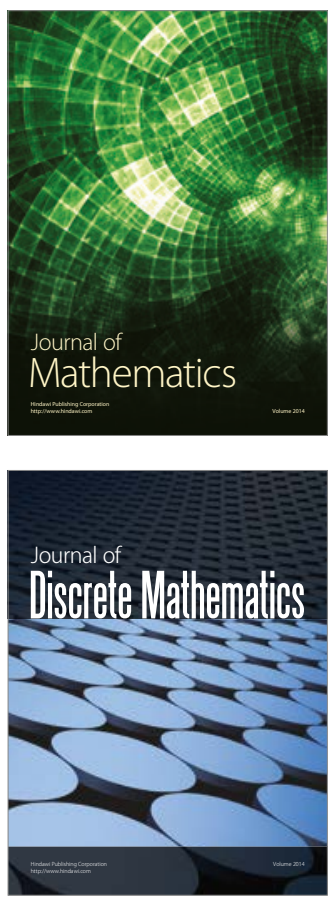

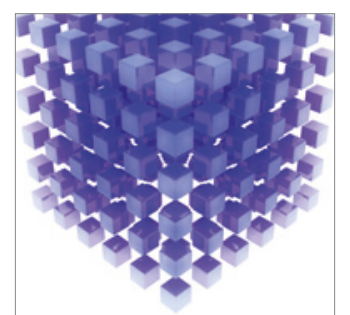

Mathematical Problems in Engineering
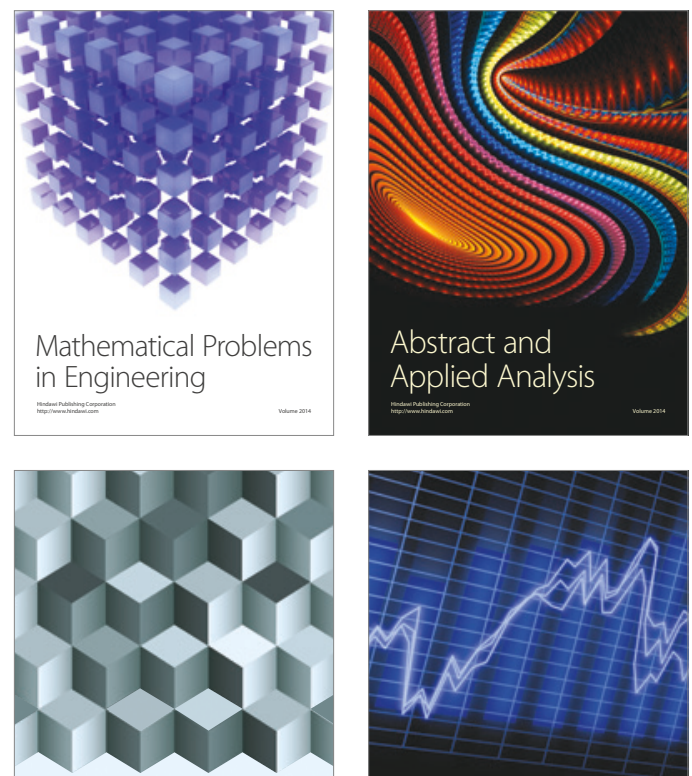

Journal of

Function Spaces

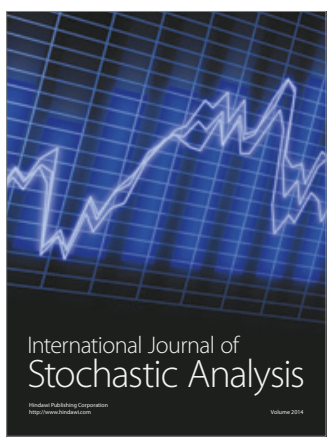

Probability and Statistics
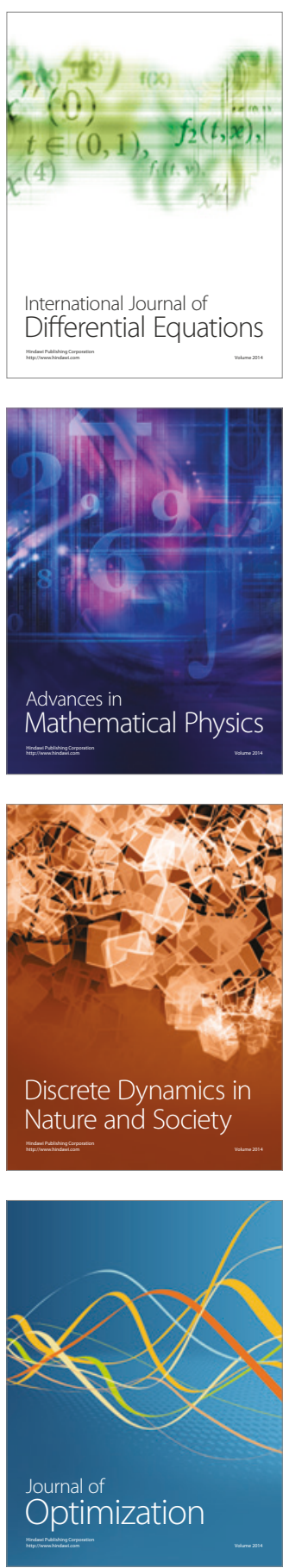